\title{
Size dependent thermodynamic properties of nanoparticles
}

\author{
S. K. Jalal ${ }^{1} *$ S. A. mawlood $^{2}$ \\ ${ }^{1}$ deptartment of General science, Charmo university \\ ${ }^{2}$ department of Physics, Salahadin university,Iraq \\ E-mail: ${ }^{1}$ sirwan.kareem@ charmouniversity.org
}

Received 6 August 2020, Revised 8 November 2020, Accepted 21 November 2020

\begin{abstract}
Surface effect and crystal structure lead to formulating a theoretical model to study the influences of size on thermodynamic parameters, such as melting temperature, Debye temperature, melting entropy and specific heat capacity, of nanoparticles. The cohesive energy as a thermodynamic quantity was used to relate the ratio of surface area to volume of nanomaterial with thermodynamic properties which depend on size of the nanomaterial. In this contribution, $\mathrm{Si}$ and $\mathrm{Au}$ nanoparticles were considered to study due to their potential applications in science and technology. It was found that melting temperature, Debye temperature, melting entropy of nanoscale size material is decreased with decreasing the size up to their critical sizes. Whereas, the specific heat capacity tends to enhance with reduction in nanoparticle size. The present results for melting temperature, melting entropy and Debye temperature are compared with experimental and theoretical observations and adequate agreements are observed.
\end{abstract}

Keywords: Nanoparticle; size; melting temperature; Debye temperature; specific heat

\section{Introduction}

The study of nanomaterial behaviors has become an inductive path of research in physics. The physical and chemical properties of bulk material depend on structure. While, nanomaterial characteristics are found not only dependent on structure, but their size and shape are often the most effective factors. The tiny size of nanomaterials leads them to exhibit different properties from their corresponding bulk form of the same material. The behavior of nanomaterials is found to depend mostly on the ratio of its surface area to the volume of the material [1,2]. Thus, the effect of the surface area of nanomaterial has a central role in determining their physical and chemical properties. As the proportion of atoms comprising a single particle is an important parameter in determining the structure of the crystal and energies of states of a material [3]. Within the range of nanoscale size, most of the atoms of the material occupy the surface, resulting in increased surface area. This factor can alter many interesting physical properties. For example, phonon density of state shift [4], an increase of elastic modulus of nanoparticles, and a decrease of melting temperature in nanomaterials are all associated with the particle size $[1,5]$.

The disparity in the state of order of melting and the lattice points refers to melting entropy. Crystalline solid behaviors such as bulk modulus, thermal expansion, melting point, and structural transformation strongly depend on melting entropy [6]. Hence, to a better understanding of the thermodynamic behaviors of nanomaterials, much importance is associated with the calculations of the melting entropy in research conducting on the phenomenon of melting. Most thermodynamic characteristics of nanomaterials such as Debye temperature, melting point, heat conductivity decrease with the decrease in sizes. Contrary, the specific heat rises when the size of nanomaterial decreased [7]. Reduction in melting point with decreasing in the size of nanoparticles size is because the nanomaterial has a high proportion of surface atoms that are less bonded to the interior atoms. The variation of the thermodynamic characteristics with size has been confirmed with experiments [8,9]. The melting temperature of $\mathrm{Au}$ nanoparticle in the range $10 \mathrm{~nm}-2 \mathrm{~nm}$ in diameter has been investigated experimentally [10].

Thermodynamic properties of nanomaterials do not only depend on size but they are also influenced by the shape of nanomaterials. For example, melting entropy, melting point, and Debye characteristic of nanofilms are measured to be greater than of nanowires and for nanowires larger than that of nanoparticles corresponding to the same material. These phenomena are well investigated by Singh [11]. Moreover, Qi [3] presented a model, analogs to equation (4), for calculating the melting temperature of nanomaterial. In this model, a shape factor is considered as an important parameter for describing the size dependence of the melting point. Several particle shapes were assumed and variation of melting temperature as a function of shape factor and at different sizes has been determined.

Several studies have been established to find the effect of altering size on the lattice parameter. Experimental and theoretical studies have proved that lattice parameter of nanoparticles varies with particle size $[11,12,32]$. Omer [13] presented a model for describing the effect of size on mean bond length in nanomaterial and developed an expression for size dependence of lattice parameter and lattice volume. Hence, the variation of relative lattice volume has been combined with a melting temperature of nanoscale particles and 
also developed a formula for bulk modulus dependence of size [13].

Moreover, Qi [1] assumed that if a particle is taken out from bulk material having the same structure to form a nanoparticle, one can visualize that the particle would have the same structure as that of its bulk counterpart, Qi [1] proposed that lattice parameter contracts for isolated nanoparticles and derived an expression for the variation of lattice parameter with size and shape. Therefore, the contraction of the lattice parameter leads to a reduction in the lattice volume of nanomaterials. Sadaiyandi [14] used the Qi [1] model to calculate the fraction of volume contraction of nanoparticles of $\mathrm{Al}, \mathrm{Au}$, and $\mathrm{Ag}$.

In the present study, the relationship between the cohesive energy and melting point is used to formulate equations for size dependence of melting point, Debye temperature, melting entropy, and heat capacity. The variation of the above thermodynamic properties for $\mathrm{Si}$ and $\mathrm{Au}$ will be the subject of this study. It has been found that most thermodynamic behavior of nanoparticles varies proportional to the inverse of the nanoparticle diameter, as a first approximation except specific heat capacity for which increases as the particle size decreases. Experimental and theoretical published data are contributed to the figures for comparison and satisfactory consistency is observed.

\section{Theoretical detail}

\subsection{Melting temperature}

It has been found that melting temperature for nanomaterials is significantly smaller than for the bulk material. Various thermodynamic models have been investigated for studying the behavior of melting entropy of nanomaterial $[13,15]$.

The cohesive energy which determines most of thermodynamic characteristics of materials [16] is a an essential thermodynamic parameter for deriving a size dependent formula of melting point, the cohesive energy of nanomaterial is the sum of the contribution of the surface atoms and the atoms in the interior, which is written as [1]:

$$
E_{\text {total }}=E_{\mathrm{o}}(n-N)+\frac{1}{2} E_{\mathrm{o}} N
$$

Where,

$\mathrm{E}_{\mathrm{o}}$ is the cohesive energy of an atom, $n$ is the number of atoms comprising the material and $N$ is the number of atoms occupied the surface. $(n-N)$ is the number of interior atoms. Eq. (1) is rewritten to express the energy per a mole:

$$
\frac{\mathrm{A} E_{\text {total }}}{n}=\mathrm{AE}_{\mathrm{o}}\left(1-\frac{N}{n}\right)+\frac{1}{2 n} \mathrm{AE}_{\mathrm{o}} N
$$

A is the Avogadro's number.

$\mathrm{A} E$ total $/ n=E_{\mathrm{n}}$ is the cohesive energy per a mole of the nanomaterial.

$\mathrm{AE}_{\mathrm{o}}=\mathrm{E}_{\mathrm{b}}$ is the cohesive energy per mole of bulk material.

Eq. (2) is arranged to form $E_{\mathrm{n}}$ :

$$
E_{\mathrm{n}}=\mathrm{E}_{\mathrm{b}}\left(1-\frac{N}{2 n}\right)
$$

The cohesive energy is proposed to be linearly related to the melting temperature [23] therefore, the relation for nanomaterials melting temperature $\mathrm{T} n$ is formulated by the following:

$$
T_{\mathrm{n}}=\mathrm{T}_{\mathrm{b}}\left(1-\frac{N}{2 n}\right)
$$

Where, $T_{b}$ is the melting point of the bulk state which is constant. N/2n depends on the size and shape of the nanomaterials. For nanoparticles $N / 2 n=2 \mathrm{~d} / D, \mathrm{~d}$ is the atomic diameter and $D$ diameter of the considered nanoparticle [1]. Therefore, from Eq. (4), the size dependence of $T_{\mathrm{n}}$ is expressed as:

$$
T_{\mathrm{n}}=\mathrm{T}_{\mathrm{b}}\left(1-\frac{2 \mathrm{~d}}{D}\right)
$$

Eq.(5) indicates that melting temperature $\left(T_{\mathrm{n}}\right)$ varies with the inverse of the diameter $\left(D^{-1}\right)$ of the nanoparticle, while $\mathrm{d}$ is the atomic diameter and depends on the structure of the material and it is size independent. Xiong [15] used Gibbs free energy for surface and bulk atoms to formulate an equation analogue to Eq. (5)

\subsection{Debye temperature}

The Debye temperature $\theta_{\mathrm{D}}$, is an important thermodynamic quantity, which appears in Debye theory of specific heat capacity of solid. Debye temperature is defined mathematically as:

$$
\theta_{\mathbf{D}}=\frac{\hbar \omega_{\mathbf{D}}}{\mathrm{K}_{\mathrm{B}}}
$$

Where $\omega_{\mathrm{D}}$ is the Debye frequency and $\mathrm{K}_{\mathrm{B}}$ is Boltzmann constant.

For nanomaterials, the amplitude of the vibration of the surface atoms is higher than that of the bulk atoms and their vibrational frequencies is smaller in comparison with that of the bulk material [4,17]. Hence, Eq. (6) predicts that $\theta_{\mathrm{D}}$ for nanomaterials decreases as the size is reduced.

Lindemann's melting theory states that crystals melt when the root mean square displacement of atoms become greater than some fractions of the interatomic spaces in the solid, which can also be valid for nanoparticles. Hence, melting temperature $T_{\mathrm{b}}$ and Debye temperature $\theta_{\mathrm{Db}}$ for bulk materials are combined as [18]:

$$
\theta_{\mathrm{Db}} \propto\left(\frac{T_{\mathrm{b}}}{\mathrm{MV}^{2 / 3}}\right)^{1 / 2}
$$

Where, $T_{\mathrm{b}}$ is the melting point of bulk state, $\mathrm{M}$ is the molecular mass, and $\mathrm{V}$ is the lattice volume per atom. Similarly nanomaterial Debye temperature $\theta_{\mathrm{Dn}}$ is:

$$
\theta_{\mathrm{Dn}} \propto\left(\frac{T_{\mathrm{n}}}{\mathrm{MV}^{2 / 3}}\right)^{1 / 2}
$$

Dividing Eq. (8) by Eq. (7) a relationship for $\theta_{\mathrm{Dn}}$ is obtained:

$\frac{\theta_{\mathrm{Dn}}}{\theta_{\mathrm{Db}}}=\left(\frac{T_{\mathrm{n}}}{\mathrm{T}_{\mathrm{b}}}\right)^{1 / 2}$

$\mathrm{T}_{\mathrm{b}}$ is constant for an assumed material. From Eq. (5) $\theta_{\mathrm{Dn}}$ is given as a function of particle diameter:

$$
\theta_{\mathrm{Dn}}=\theta_{\mathrm{Db}}\left(1-\frac{2 \mathrm{~d}}{D}\right)^{1 / 2}
$$


Eq. (10) is used in the current study to evaluate the size dependent $\theta_{\mathrm{Dn}}$ for $\mathrm{Si}$ and $\mathrm{Au}$ nanoparticles.

\section{3 melting entropy}

The melting entropy for nanomaterials has got intensive attention by researchers [6]. The vibrational entropy of melting $\left(\mathrm{S}_{\mathrm{vib}}\right)$ for bulk crystals in relating to the melting temperature, is given below [15]:

$$
S_{\mathrm{vib}}=\frac{3 \mathrm{R}}{2} \ln \left(\frac{\mathrm{T}_{\mathrm{b}}}{\mathrm{C}}\right)
$$

Where, $\mathrm{C}$ is constant and $R$ is the gas constant. It has been stated that the nature of melting of metallic crystals is vibrational [19] .Therefore, the $\left(S_{\text {vib }}\right)$ can be equal to $\left(S_{\mathrm{m}}\right)$. Thus, is rewritten for bulk material as follow:

$S_{\mathrm{mb}}=\frac{3 \mathrm{R}}{2} \ln \left(\frac{T_{\mathrm{b}}}{\mathrm{C}}\right)$

And for nanomaterials

$$
S_{\mathrm{mn}}=\frac{3 \mathrm{R}}{2} \ln \left(\frac{T_{\mathrm{n}}}{\mathrm{C}}\right)
$$

$S_{\mathrm{mn}}$ is the melting entropy of nanomaterials. Logarithm rules allow relating Eqs. (12) - (13) as following:

$$
S_{\mathrm{mn}}=\mathrm{S}_{\mathrm{mb}}+\frac{3 \mathrm{R}}{2} \ln \left(\frac{T_{\mathrm{n}}}{\mathrm{T}_{\mathrm{b}}}\right)
$$

It can be deduced from Eq. (14) that $S_{\mathrm{mn}}$ of a nanomaterial is directly linked to its size dependent melting point $T_{\mathrm{n}}$. To calculate $S_{\mathrm{mn}}$ and from Eq. (5) we get the following equation:

$$
S_{\mathrm{mn}}=\mathrm{S}_{\mathrm{mb}}+\frac{3 \mathrm{R}}{2} \ln \left(1-\frac{2 \mathrm{~d}}{D}\right)
$$

The melting entropy model given in Eq. (15) is derived to apply on nano crystals for which the effects of electronic melting entropy has vanished. To consider semiconductors, the model is modified by Safaei [6] in which the contributions of electronic melting entropy have been taken into account in the expression of total melting entropy.

\subsection{Specific heat capacity}

An important thermodynamic quantity which is expected to vary under the influence of changing size of nanomaterials is the heat capacity. Specific heat is the amount of energy supplied to rise the temperature of a unit mass of a material by one degree of Kelvin. In bulk form, the specific heat capacity of a material depends only on temperature. However, due to the surface effect, the specific heat of nanomaterials differs significantly from the bulk state of the same material.

Many experimental and theoretical studies have been performed to investigate the effect of size on specific heat. The specific heat capacity of $\mathrm{Pd}$ with the size of $6 \mathrm{~nm}$ and at constant pressure is measured and found that its value is higher than its bulk state value by around of $40 \%$ [20].This implies that in the range of nanoscale size the specific heat capacity varies with both temperature and size. An experiment on small lead nanoparticles with sizes $2.2 \mathrm{~nm}$ and $3.7 \mathrm{~nm}$ at low temperature was carries out and showed significant enhancement in the values of heat capacity [21]. A relationship between specific heat of bulk material $C_{p b}$ and Debye temperature at constant pressure is obtained, on the basis of Debye's theory [7]:

$$
C_{\mathrm{pb}} \propto \frac{1}{\theta_{\mathrm{Db}}^{2}}
$$

Similarly, for nanomaterials, the expression is:

$$
C_{\mathrm{pn}} \propto \frac{1}{\theta_{\mathrm{Dn}}^{2}}
$$

Eq. (16) is divided by Eq. (17) to get this form:

$C_{\mathrm{pn}}=\mathrm{C}_{\mathrm{pb}}\left(\frac{\theta_{\mathrm{Dn}}}{\theta_{\mathrm{Db}}}\right)^{-1 / 2}$

$\mathrm{C}_{\mathrm{pn}}$, is the specific heat of nanomaterial. Substituting Eq. (10) in to Eq. (18), The size dependence of $\mathrm{C}_{\mathrm{pn}}$ is obtained in the following relation:

$C_{\mathrm{pn}}=\mathrm{C}_{\mathrm{pb}}\left(1-\frac{2 \mathrm{~d}}{D}\right)^{-1}$

Eq. (19) represents the nanomaterial specific heat in terms of its bulk specific heat and as a function of size. For nanomaterials, $C_{\mathrm{pn}}$ is predicted to rise with decreasing size.

\begin{tabular}{|c|c|c|c|c|c|}
\hline Elements & $\mathrm{s} \quad \mathrm{d}(\mathrm{nm})[5]$ & $\mathrm{S}_{\mathrm{mb}}$ & $\theta_{D}(K)$ & $\mathrm{T}_{\mathrm{mb}}(\mathrm{K})[5]$ & $\mathrm{C}_{\mathrm{pb}}$ \\
\hline $\mathrm{Si}$ & 0.3368 & 30 & $645[22]$ & 1685 & 712 [23] \\
\hline $\mathrm{Au}$ & 0.288 & 9.34 & 184 [25] & 1337 & $129[26]$ \\
\hline
\end{tabular}
The enhancement in specific heat at small sizes is caused by a high contribution of the surface atoms of the atomic thermal vibrational energies.

Table 1. Input Parameters used in the calculations. $\mathrm{S}_{\mathrm{m}}$ is measured in unit of $(\mathrm{J} / \mathrm{mol} . \mathrm{K})$ and $C_{p b}$ in unit $(\mathrm{J} / \mathrm{kg} . \mathrm{C})$

\subsection{Results and discussion}

The required parameters in this study are presented in Table1. The important thermodynamic properties of nanoscale materials, such as melting point $T_{n}$, Debye temperature $\theta_{D n}$, melting entropy $S_{m n}$ and specific heat capacity $C_{p n}$ for $S i$ and $\mathrm{Au}$ have been studied.

Eq. (5) was used to evaluate the variation of melting point with decreasing the diameter $D$ of nanoparticles. Figures 1-2 indicates that melting temperature for $\mathrm{Si}$ and $\mathrm{Au}$ nanoparticle drops with the decrease the in the size of the nanoparticle. From the melting curves, two main trends can be observed. For diameters $D$ larger than $10 \mathrm{~nm}$, melting temperature decreases steadily with altering size and the curves are appeared nearly invariant. However, in contrast, the effect of size is very different where $D<10 \mathrm{~nm}$. Melting temperature drops rapidly with a slight decrease in the particle size below the limit of $10 \mathrm{~nm}$. For both considered nanoparticles, when the diameter is reduced to around $1.5 \mathrm{~nm}$ the trend of melting point declines to about half of the bulk value.

Declining in the values of melting point is caused by a high proportion of the surface atoms contributed from ratio of the surface area to volume. The high surface to volume ratio indicated breaking more bonds, since the surface atoms experience a less interaction with their neighbors than do the interior atoms.

The present results of melting point of Si and Au have been compared with experimental and theoretical data, and substantial agreements can be observed with all results. Regarding to $T_{\mathrm{n}}$ values of Au nanoparticle in the Figure 2, a better accord with experimental data than that of $\mathrm{Si}$ value is observed for the same size. This can be attributed that $\mathrm{Au}$ is a metal and cohesive energy model well established for metals. 


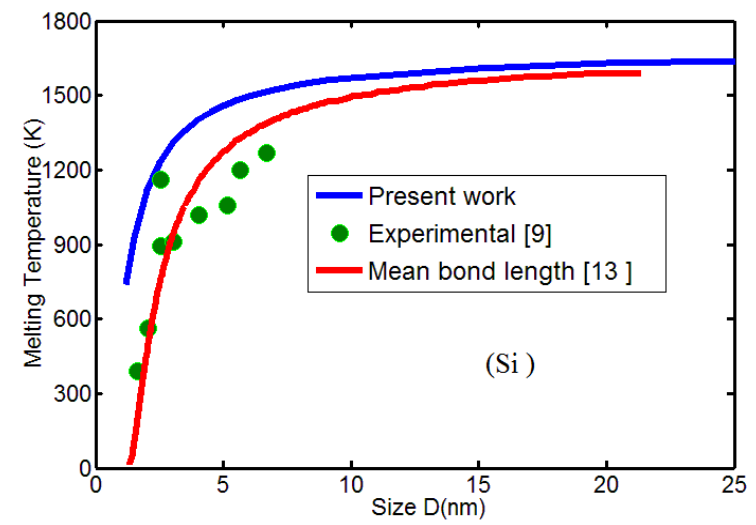

Figure 1. Melting temperature $\left(T_{n}\right)$ at different size calculated for Si nanoparticle. Experimental data [9] and theoretical data [13] are reported.

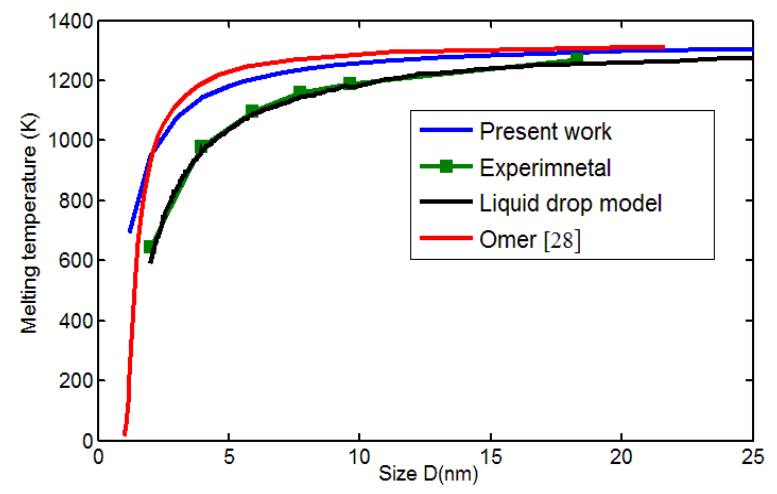

Figure 2. Melting temperature $\left(T_{n}\right)$ at different size calculated for $A u$, compared with the experimental data[10],liquid drop model[27] and mean bonding length model Omer[28].

Figures 3- 4 represent the results of size dependence of Debye temperature $\theta_{\text {Dn }}$ for $\mathrm{Si}$ and $\mathrm{Au}$ nanoparticles calculated using Eq. (10). It is observed that the $\theta_{\text {Dn }}$ reduces non-linearly with the decrease in the diameter of the particles. One can find that the $\theta_{\text {Dn }}$ alters very slowly with the particle's dimension when values of the diameter $D$ are greater than $10 \mathrm{~nm}$. After this limit, Debye temperature drops sharply for both $\mathrm{Si}$ and $\mathrm{Au}$ nanoparticles considered to study. This trend is attributed that as the nanoparticles size decreases the frequency of vibrations drops and Debye temperature varies with Debye frequency [6].

Theoretical data of $\theta_{\mathrm{Dn}}$ for $\mathrm{Si}$ in Figure 3 and experimental data of $\theta_{\mathrm{Dn}}$ for $\mathrm{Au}$ in Figure 4 have been reported. It can be noticed that our result for $\mathrm{Au}$ fits well with the experimental data. On the other hand, the theoretical data for Si nanoparticle reported in [29] predicts a sharp drop in the values of $\theta_{\text {Dn }}$, in which $\theta_{\text {Dn }}$ values approach zero at the diameter of around $1 \mathrm{~nm}$. While in the present model the results of $\theta_{\mathrm{Dn}}$ decreases significantly but do not approach to zero. In addition, the curve of experimental data of $\theta_{\mathrm{Dn}}$ for $\mathrm{Au}$ in Figure 4 , show that $\theta_{\mathrm{Dn}}$ does not approach to zero even at lowest value of the diameter (D) and this confirms our results of $\theta_{\mathrm{Dn}}$ calculated with Eq. (14). On the other hand, the literature data of $\theta_{\mathrm{Dn}}$ for $\mathrm{Si}$ has been calculated based on the mean bonding length variation in which lattice volume is expanded with decreasing nanoparticle size [29].

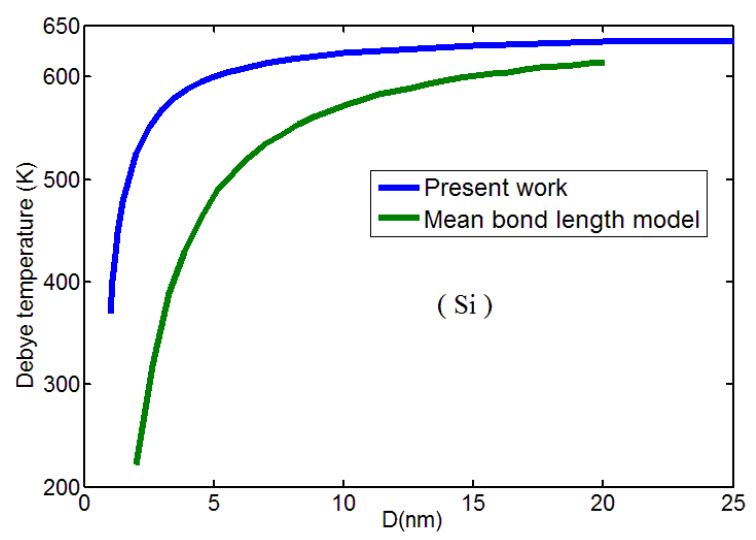

Figure 3. Size dependence of Debye temperature $\theta_{D n}$ for $\mathrm{Si}$, calculated in the present work and mean bond length model data is reported[29].

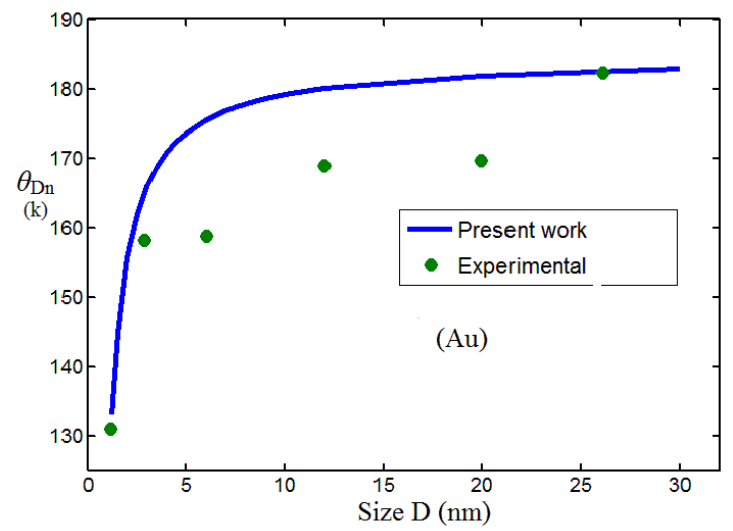

Figure 4. Size dependence of $\theta_{D n}$ for Au nanoparticles, calculated in the present work. in comparison with Experimental data[10].

This model is expanded further to investigate the size dependence of melting entropy $S_{m n}$ of the nanoparticles. Eq. (15) predicts that melting entropy drops with the decrease in particle diameter. Figures 5-6 illustrate the variation of $\mathrm{S}_{\mathrm{mn}}$ as a function of diameter for both Silicon and Gold nanoparticles. For the purpose of comparison, the theoretical data for Si nanoparticles investigated by Safaei [6] is presented in Figure5. Safaei determined the size dependence of $S_{m n}$ for $\mathrm{Si}$ by using the theory of size dependence of electronic components of melting entropy and its contribution on the overall melting entropy of nano semiconductors. In Safaei model [6], the contributions of electrons have been subtracted from the value of melting entropy calculated with Eq. (15). In Figure 5 the present results for melting entropy of Si calculated with Eq. (15) shows a satisfactory agreement with the data of Safai [6] for considering the effects of electron melting entropy, and there is only a very little divergence is observed with the present work. Therefore, Safai data [6] supports our results, and implies that the present model which derived for metals could also be used for nano semiconductors.

Moreover, for calculating the above thermodynamic parameters $T_{\mathrm{n}}, \theta_{\mathrm{Dn}}$ and $S_{\mathrm{mn}}$, the critical diameter of the nanoparticle is important. The critical size (diameter) is $D$ $=3 \mathrm{~d}$ [13] for nanoparticle. Depending on the atomic diameter, the critical size for $\mathrm{Si}$ and $\mathrm{Au}$ are $1.01 \mathrm{~nm}$, and $\mathrm{Au}$ $0.86 \mathrm{~nm}$ respectively. 


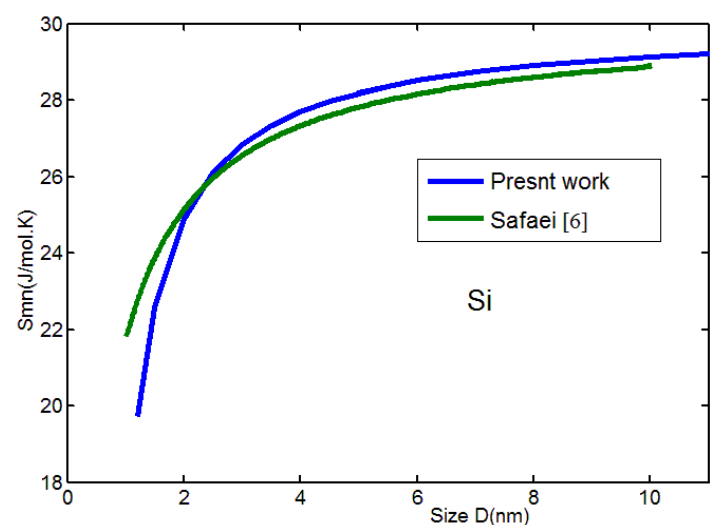

Figure 5. Variation of melting entropy $S_{m n}$ with size for $\mathrm{Si}$, compared with theoretical data [6].

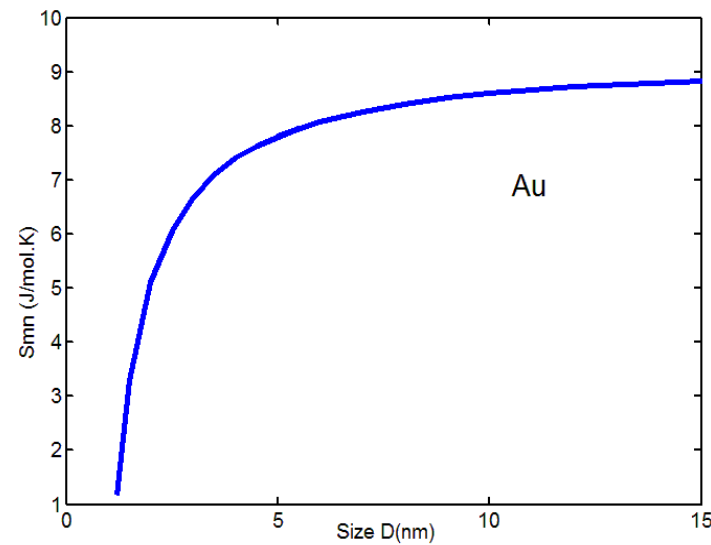

Figure 6. Variation of melting entropy $S_{m n}$ with size for $\mathrm{Au}$ nanoparticle.

Further interesting investigation, is the understanding of specific heat capacity dependence on nanoparticle size. Apart from the above studied thermodynamic quantities, it is expected from Eq.(19), that as the size of the nanomaterial is decreased the specific heat capacity $C_{\mathrm{pn}}$ tends to increase. Figure 6 shows how the $C_{\mathrm{pn}}$ of $\mathrm{Si}$ and $\mathrm{Au}$ nanoparticle increases with the reduction in their diameters. It is shown that $C_{\mathrm{pn}}$ increases very slightly when the size decreases from $30 \mathrm{~nm}$ to $10 \mathrm{~nm}$. While, as the size decreased further from $10 \mathrm{~nm}$ towards $2 \mathrm{~nm}$, the curves of the specific heat rose dramatically to around double value of that of the bulk state. Specific heat of nanomaterial is found to depend on lattice vibrations [30]. There are two vibrations contribute to the specific heat capacity of nanomaterials, the interior atom vibrations and an additional surface atom vibrations[31].

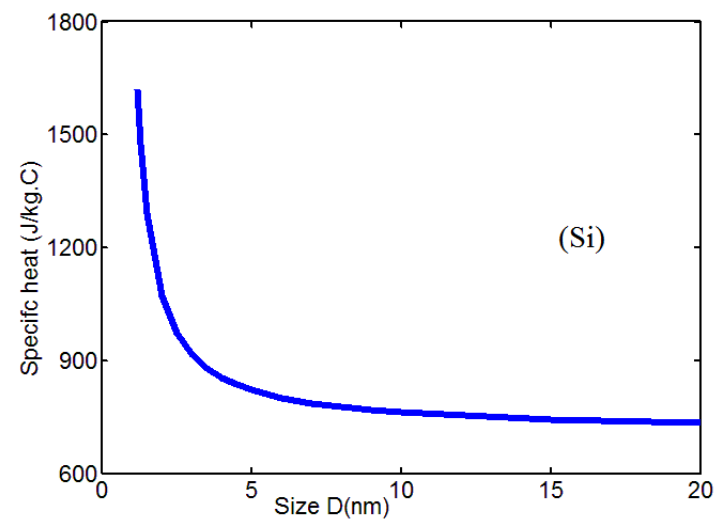

Figure 7. Size dependence of specific heat $\mathrm{C}_{\mathrm{pn}}$ for $\mathrm{Si}$, nanoparticle.

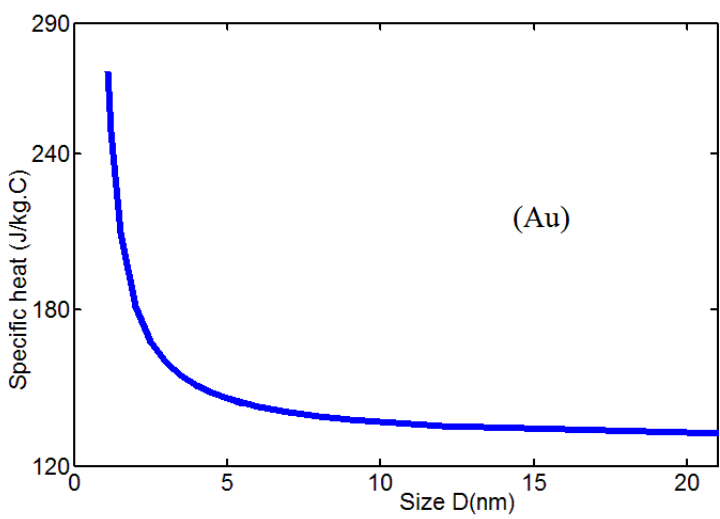

Figure 8. Size dependence of specific heat $C_{\mathrm{pn}}$ for $\mathrm{Au}$, nanoparticle.

\subsection{Conclusion}

This theoretical study is an attempt for understanding some thermodynamic parameters of nanoparticles which exhibit to depend on particle size. It is concluded that $T_{\mathrm{n}}$, $\theta_{\mathrm{Dn}}$ and $\mathrm{S}_{\mathrm{mn}}$ for nanoparticles are decreased with decreasing their sizes up to their critical values. In contrast, the specific heat capacity $C_{\mathrm{pn}}$ is the only thermodynamic quantity which increases with the decrease in size. Decreasing size above $5 \mathrm{~nm}$ causes all considered thermodynamic quantities to change steadily. On the other hand, very drastic variations happen when sizes approach $2 \mathrm{~nm}$. Present results show good agreements with available experimental and other theoretical data that have been included for the purpose of comparison, and they also confirm the validity of this model. The result in Figure 5 for $\mathrm{Si}$ melting entropy in comparison with the theoretical published data [6], confirm that Eq. (15) which developed for metals can be applicable for semiconductors since the effect of electronic melting entropy is considerably small.

\section{References:}

[1] W. Qi, "Size effect on melting temperature of nanosolids," Physica B: Condensed Matter, vol. 368, no. 1-4, pp. 46-50, 2005.

[2] W. Qi and M. Wang, "Size and shape dependent lattice parameters of metallic nanoparticles," Journal of Nanoparticle Research, vol. 7, no. 1, pp. 51-57, 2005.

[3] W. Qi and M. Wang, "Size and shape dependent melting temperature of metallic nanoparticles," Materials chemistry and physics, vol. 88, no. 2-3, pp. 280-284, 2004.

[4] R. Meyer and D. Comtesse, "Vibrational density of states of silicon nanoparticles," Physical Review B, vol. 83, no. 1, p. 014301, 2011.

[5] M. Omar, "Structural and Thermal Properties of Elementary and Binary Tetrahedral Semiconductor Nanoparticles," International Journal of Thermophysics, vol. 37, no. 1, p. 11, 2016.

[6] A. Safaei and M. A. Shandiz, "Melting entropy of nanocrystals: an approach from statistical physics," Physical Chemistry Chemical Physics, vol. 12, no. 47, pp. 15372-15381, 2010. 
[7] Y. Zhu, J. Lian, and Q. Jiang, "Modeling of the melting point, Debye temperature, thermal expansion coefficient, and the specific heat of nanostructured materials," The Journal of Physical Chemistry C, vol. 113, no. 39, pp. 16896-16900, 2009.

[8] M. Cottie, "The weird world of nanoscale," University of Technology, Sydney, PO Box, vol. 123.

[9] C. Yang, M. Xiao, W. Li, and Q. Jiang, "Size effects on Debye temperature, Einstein temperature, and volume thermal expansion coefficient of nanocrystals," Solid state communications, vol. 139, no. 4, pp. 148-152, 2006.

[10] P. Buffat and J. P. Borel, "Size effect on the melting temperature of gold particles," Physical review A, vol. 13, no. 6, p. 2287, 1976.

[11] M. Singh, S. Lara, and S. Tlali, "Effects of size and shape on the specific heat, melting entropy and enthalpy of nanomaterials," Journal of Taibah University for Science, vol. 11, no. 6, pp. 922-929, 2017.

[12] P. Montano, W. Schulze, B. Tesche, G. Shenoy, and T. Morrison, "Extended x-ray-absorption finestructure study of Ag particles isolated in solid argon," Physical review B, vol. 30, no. 2, p. 672, 1984.

[13] M. Omar, "Models for mean bonding length, melting point and lattice thermal expansion of nanoparticle materials," Materials Research Bulletin, vol. 47, no. 11, pp. 3518-3522, 2012.

[14] K. Sadaiyandi, "Size dependent Debye temperature and mean square displacements of nanocrystalline $\mathrm{Au}$, Ag and Al," Materials Chemistry and Physics, vol. 115, no. 2-3, pp. 703-706, 2009.

[15] S. Xiong, W. Qi, Y. Cheng, B. Huang, M. Wang, and Y. Li, "Universal relation for size dependent thermodynamic properties of metallic nanoparticles," Physical Chemistry Chemical Physics, vol. 13, no. 22, pp. 10652-10660, 2011.

[16] J. H. Rose, J. Ferrante, and J. R. Smith, "Universal binding energy curves for metals and bimetallic interfaces," Physical Review Letters, vol. 47, no. 9, p. 675, 1981.

[17] C. Q. Sun, Y. Wang, B. Tay, S. Li, H. Huang, and Y. Zhang, "Correlation between the melting point of a nanosolid and the and the cohesive energy of a surface atom," The Journal of Physical Chemistry $B$, vol. 106, no. 41, pp. 10701-10705, 2002.

[18] J. Dash, "History of the search for continuous melting," Reviews of Modern Physics, vol. 71, no. 5, p. $1737,1999$.

[19] A. R. Ubbelohde, Melting and crystal structure. Clarendon press, 1965.

[20] J. Rupp and R. Birringer, "Enhanced specific-heatcapacity (c p) measurements (150-300 K) of nanometer-sized crystalline materials," Physical Review B, vol. 36, no. 15, p. 7888, 1987.
[21] V. Novotny, P. Meincke, and J. Watson, "Effect of size and surface on the specific heat of small lead particles," Physical Review Letters, vol. 28, no. 14, p. 901, 1972.

[22] O. Madelung, U. Rössler, and M. Schulz, "Silicon (Si), Debye temperature, heat capacity, density, hardness, melting point," Landolt-Börnstein Substance/Property Index, vol. 41, pp. 1-16, 2002.

[23] A. Okhotin, A. Pushkarskii, and V. Gorbachev, "Thermophysical Properties of Semiconductors, Atom Publ," House, Moscow, 1972.

[24] E. Steigmeier, "The Debye temperatures of Iii-V compounds," Applied Physics Letters, vol. 3, no. 1, pp. 6-8, 1963.

[25] A. Balerna and S. Mobilio, "Dynamic properties and Debye temperatures of bulk Au and Au clusters studied using extended $\mathrm{x}$-ray-absorption fine-structure spectroscopy," Physical Review B, vol. 34, no. 4, p. 2293, 1986.

[26] A. I. Oliva and J. Lugo, "The physical properties of nanomaterials: A challenge in materials science," in 2015 12th International Conference on Electrical Engineering, Computing Science and Automatic Control(CCE), 2015, pp. 1-6.

[27] K. Nanda, S. Sahu, and S. Behera, "Liquid-drop model for the size-dependent melting of lowdimensional systems," Physical review A, vol. 66, no. 1, p. 013208, 2002.

[28] M. Omar, "Critical Size Structure Parameters for Au Nanoparticles," in Advanced Materials Research, 2013, vol. 626, pp. 976-979.

[29] A. K Suleiman "Optical and Acoustic Gruneisen Parameter of Nano-sized Particles.” Eurasian Journal of Science and Engineering 4, 19.

[30] B.-X. Wang, L.-P. Zhou, and X.-F. Peng, "Surface and size effects on the specific heat capacity of nanoparticles," International journal of thermophysics, vol. 27, no. 1, pp. 139-151, 2006.

[31] L. Rivière, N. Caussé, A. Lonjon, É. Dantras, and C. Lacabanne, "Specific heat capacity and thermal conductivity of PEEK/Ag nanoparticles composites determined by Modulated-Temperature Differential Scanning Calorimetry," Polymer degradation and stability, vol. 127, pp. 98-104, 2016.

[32] S. K. Jalal, A. M. Al-Sheikh, R. H. Al-Saqa,"high pressure effects of phonon spectrum of silicon nanoparticle"Iranian Journal of Science and Technology, Transactions A: Science. forthcoming. 